\title{
Morphometry of the Orbital Region Soft Tissues in Down Syndrome
}

\author{
Chiarella Sforza, MD, PhD, * Fadil Elamin, MSc, BSc, BDS, MOrthEd, FFDRCSI, $\dagger$ \\ Claudia Dellavia, DDS, PhD, * Riccardo Rosati, DDS, PhD, * Gianluigi Lodetti, DDS, ** \\ Andrea Mapelli, MBioEng, * and Virgilio Ferruccio Ferrario, MD, PhD*
}

\begin{abstract}
The orbital region of subjects with Down syndrome (DS) has been scanty described so far. We wanted to detail the morphologic characteristics of the soft tissue orbital region in Italian and North Sudanese subjects with DS.

The three-dimensional coordinates of 10 landmarks on the orbital soft tissues were obtained using computerized anthropometry in 53 Italian and 64 North Sudanese subjects with DS aged 4 to 52 years, and in 461 (Italian) and 682 (North Sudanese) sex- and age-matched controls. From the landmarks, linear distances, ratios, areas, and angles were calculated, $z$ scores computed, and compared by $t$-tests and analyses of covariance.

In North Sudanese DS subjects, intercanthal width and height-tolength ratio were increased; biorbital width, eye height, length, and area were reduced. Eye fissure and orbital inclinations relative to Frankfort plane were reduced, whereas orbital inclinations versus the true horizontal were increased. In Italian DS men, orbital height and height-to-length ratio were increased, eye length was decreased; orbital inclination versus the true horizontal was increased. For almost all measurements, a significant effect of age was found. No effects of sex were found. Ethnic group influenced orbital height, area, and orbital inclination versus Frankfort plane. All paired measurements had similar discrepancies on both sides.

The orbital soft tissues of North Sudanese DS subjects differed from those of their reference subjects, but this was only partially true for Italian subjects. The 2 ethnic groups had different alterations in their soft tissue orbital regions that were influenced by age, but not by sex.
\end{abstract}

From the *Functional Anatomy Research Center, Laboratorio di Anatomia Funzionale dell'Apparato Stomatognatico, Dipartimento di Morfologia Umana e Scienze Biomediche "Città Studi," Facoltà di Medicina e Chirurgia, Università degli Studi di Milano, Milano, Italy; †Department of Orthodontics, El-Razi College Dentistry, Khartoum, Sudan; and tDipartimento di Specialità Chirurgiche, Scienze Radiologiche e Medico Forensi, Università degli Studi di Brescia, Italy.

Received December 18, 2010.

Accepted for publication April 2, 2011.

Address correspondence and reprint requests to Dr. Chiarella Sforza, Dipartimento di Morfologia Umana e Scienze Biomediche

"Città Studi," via Mangiagalli 31, I-20133 Milano, Italy;

E-mail: Chiarella.Sforza@unimi.it

The authors report no conflicts of interest.

Copyright (C) 2012 by Mutaz B. Habal, MD

ISSN: $1049-2275$

DOI: $10.1097 /$ SCS.0b013e3182418eba
Key Words: Face, three-dimensional, soft tissues, anthropometry, Down syndrome

(J Craniofac Surg 2012;23: 198-202)

The soft tissues of the orbital region play a major role in the evaluation and recognition of the craniofacial complex, and relevant anthropometric data are often used for multiple diagnostic and forensic procedures. Among others, there are evaluations of traumas, chromosomal and single gene alterations, teratogenic-induced conditions such as the fetal alcohol syndrome, and facial reconstruction. ${ }^{1-3}$ Sex, age, and ethnicity are factors that influence the soft tissue characteristics of the orbital region significantly, both in healthy subjects and in patients. $^{2-7}$

Down syndrome (DS) (Mendelian Inheritance in Man no. 1906858) is the most frequent autosomal aneuploidy in live-born humans. Down syndrome is produced by the complete or partial trisomy of chromosome 21 , and it is characterized by several morphologic and functional alterations of body structures, from cellular organelles to multiorgan systems, which are present in varying degrees in the affected individuals. ${ }^{8}$ A distinctive craniofacial appearance is present in almost all persons with DS. ${ }^{8}$

Subjects with DS have a reduced head size with a modified head shape; a decrease in the interorbital distance, a small palpebral fissure, and a prominent forehead; a hypoplastic facial middle third (maxilla) with a less prominent nose; a reduced facial lower third with a prominent mandible; and smaller ears. ${ }^{9-21}$ Most of these characteristics were described in white subjects. ${ }^{9-15,18,19}$ In contrast, scanty data on the craniofacial phenotype of African subjects with DS have been published, and only the ears and nasolabial regions have been detailed so far. ${ }^{20,21}$

Among the various parts of the face, the orbital region has been insufficiently studied in postnatal subjects with DS. Scanty data were provided by Farkas et $\mathrm{al}^{10-13}$ and Bagic and Verzak ${ }^{9}$ whereas recent investigations analyzed orbital skeletal measurements in normal and DS fetuses. ${ }^{16,17}$ Development of the structures of the upper facial third is strictly related to forebrain development, and alterations in orbital characteristics may derive from cerebral malformations in the embryonic and fetal period. ${ }^{1,22}$

In the current study, the faces of 2 groups of subjects with DS (Italian white and North Sudanese) were imaged in 3 dimensions using computerized instruments, and the morphologic features of their orbital soft tissues were quantitatively analyzed using indirect, computerized anthropometry. Data were compared with values collected in normal, healthy individuals of the same age, sex, and ethnicity. We wanted to assess if (1) subjects with DS have different characteristics of their soft tissue orbital region when compared with 
normal; (2) the 2 ethnic groups have different alterations in their soft tissue orbital regions; (3) the alterations differ between men and women; (4) the alterations change with increasing age.

\section{MATERIALS AND METHODS}

\section{Subjects}

One hundred seventeen subjects with DS from 2 different ethnic groups were included in the current study. The Italian subjects were 19 women aged 16 to 45 years (mean, 27 [SD, 7] years) and 34 men aged 13 to 52 years (mean, 27 [SD, 9] years); they all were athletes attending the Italian games of Special Olympics; details about these subjects were previously published. ${ }^{14,15,18,19}$ The Northern Sudanese subjects were 18 women aged 5 to 34 years (mean, 15 [SD, 9] years) and 46 men aged 4 to 33 years (mean 15 [SD, 7] years); they all attended special needs schools in Khartoum (Sudan); details about these subjects were previously published. ${ }^{20,21}$ None of the subjects had undergone craniofacial surgeries.

Age- and sex-matched control subjects were recruited for both ethnic groups: 461 Italian control subjects (118 women, 343 men) and 682 North Sudanese control subjects (343 women, 339 men). These subjects had no previous history of craniofacial trauma or congenital anomalies. The reference groups were collected from subjects and staff attending preschools, schools, and universities in Northern Italy and in Khartoum State.

All the analyzed individuals and the parents/legal guardians of the DS subjects and of all the reference subjects younger than 18 years gave their written informed consent to the study after explanation of the nature of the study. All procedures were not invasive; did not provoke damages, risks, or discomfort to the subjects; were performed according to the tenets of the Declaration of Helsinki; and were preventively approved by the local ethic committees.

\section{Collection of Three-Dimensional Facial Landmarks}

A detailed description of data collection can be found elsewhere. ${ }^{7,14,15,18-21}$ In brief, for each Italian subject, a single experienced operator located a set of 50 landmarks (distributed on the head, face, orbits, nose, lips and mouth, ears) and marked them on the cutaneous surface. Three-dimensional $(x, y, z)$ coordinates of the facial landmarks were obtained with a computerized electromechanic digitizer (Microscribe G2; Immersion Corporation, San Jose, CA) with an accuracy of $0.38 \mathrm{~mm}$ (workspace $50^{\prime \prime}$ sphere). The reproducibility of landmark identification and marker positioning were previously reported and found to be reliable, with a mean technical error of measurement of $1.8 \mathrm{~mm}$ and no statistically significant systematic errors. ${ }^{23}$ The data collection procedure with the electromechanic instrument gave a mean technical error of measurement of $1.33 \mathrm{~mm}^{14}$

For the North Sudanese subjects, a portable handheld laser scanner (FastSCAN Cobra; Polhemus Inc, Colchester, VT) was used for data collection, and actual anthropometric calculations were performed off-line. The technique produces a detailed threedimensional model of the face. The reported precision of the laser scanner is approximately $0.5 \mathrm{~mm}$, and time of exposure from ear to ear, trichion to menton scan, is 20 to 30 seconds. The files of the three-dimensional facial scans were obtained and stored on magnetic media. Off-line, the three-dimensional coordinates of the same set of 50 standardized landmarks were identified on the digital facial reconstruction. The reproducibility of facial digitization and landmark identification were previously reported and found to be reliable, with a mean technical error of measurement of $0.755 \mathrm{~mm}$, and there were no statistically significant systematic errors. ${ }^{20,21}$
For both ethnic groups, among the 50 soft tissue landmarks that were identified, the following paired soft tissue landmarks were used in the current analysis (right and left side noted $r$ and l): $\mathrm{ex}_{\mathrm{r}}, \mathrm{ex}_{1}$, exocanthion; $\mathrm{en}_{\mathrm{r}}, \mathrm{en}_{\mathrm{l}}$, endocanthion; $\mathrm{or}_{\mathrm{r}}$, or $_{\mathrm{l}}$, orbitale; $_{\mathrm{os}}, \mathrm{os}_{\mathrm{l}}$, orbitale superius; $t_{r}, t_{1}$, tragion (Fig. 1 ). calculations.

Custom computer programs were used for all the subsequent

\section{Data Analysis}

The three-dimensional coordinates of the landmarks obtained on each subject were used to calculate the following measurements ${ }^{7}$ :

- linear distances (unit: millimeters): biorbital width $\left(\mathrm{ex}_{\mathrm{r}}-\mathrm{ex}_{1}\right)$, intercanthal width $\left(\mathrm{en}_{\mathrm{r}}-\mathrm{en}\right)$, right and left height of the orbit (os-or), right and left length of the eye fissure (en-ex);

- ratios (unit: percentage): right and left height of the orbit to length of the eye fissure ratio (os-or / en-ex $\times 100$ );

- angles (unit: degrees): right and left inclination of the eye fissure (angle of the en-ex line vs the true horizontal, head in natural head position), right and left inclination of the orbit (angle of the os-or line versus the true horizontal, head in natural head position), right and left inclination of the orbit relative to Frankfort plane (angle between the os-or and t-or lines);

- areas (unit: millimeters squared): right and left external orbital surface area (area of the quadrangle between ex, os, en, and or).

In the control individuals, descriptive statistics were calculated for each variable separately for each ethnic group, age, and sex. The individual measurements obtained in the 117 subjects with DS were transformed to $z$ scores by subtracting each from its ethnic, sex, and age reference mean value and dividing by the relevant reference SD. ${ }^{9-13,17,20,21}$

\section{Statistical Calculations}

For each measurement, descriptive statistics (mean and SD) were computed for the values of the $z$ scores separately for Italian and North Sudanese persons and for men and women.

Three kinds of statistical comparisons were made: at first, paired Student's $t$-tests allowed to assess the significance of the $z$ scores, that is, if the measurements obtained in the subjects with DS actually differed from the measurements obtained in control subjects (null hypothesis: the $z$ scores should be 0 if the values in DS subjects do not differ from the controls matched for ethnicity, sex, and age; alternative hypothesis: $z$ scores are significantly different from 0 ).

Subsequently, to detect age-related differences in the discrepancies of the DS subjects from the controls, ${ }^{1}$ linear regression analyses were performed between age and the $z$ scores, separately for men and women and for the ethnic groups.

Lastly, to investigate if the discrepancies between control subjects and subjects with DS were the same or differed in the 2 ethnic groups, or in the 2 sexes, or in the 2 sides (the last, only for paired measurements), multifactorial mixed-design analyses of covariance (ANCOVAs) were performed, using age as covariate and

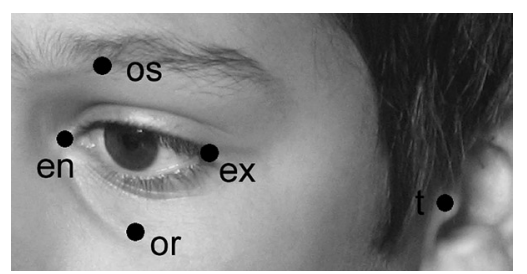

FIGURE 1. Digitized three-dimensional soft tissue orbital landmarks used in the current study. Ex indicates exocanthion; en, endocanthion; or, orbitale; os, orbitale superius; $t$, tragion. 
TABLE 1. Three-Dimensional Soft Tissue Orbital Morphometry in Subjects With DS as Compared With Control Subjects

\begin{tabular}{|c|c|c|c|c|c|c|c|c|c|c|c|c|c|c|c|c|}
\hline \multirow[b]{3}{*}{ Measurement } & \multicolumn{8}{|c|}{ Italy } & \multicolumn{8}{|c|}{ North Sudan } \\
\hline & \multicolumn{4}{|c|}{ Men $(n=34)$} & \multicolumn{4}{|c|}{ Women $(n=19)$} & \multicolumn{4}{|c|}{$\operatorname{Men}(n=46)$} & \multicolumn{4}{|c|}{ Women $(n=18)$} \\
\hline & Mean & SD & $P^{*}$ & $r \dagger$ & Mean & SD & $P^{*}$ & $r \dagger$ & Mean & SD & $P^{*}$ & $r \dagger$ & Mean & SD & $P^{*}$ & $r \dagger$ \\
\hline $\mathrm{ex}_{\mathrm{r}}-\mathrm{ex}_{1}$ & -0.462 & 1.358 & NS & NS & -0.675 & 2.008 & NS & $0.621 \%$ & -1.649 & 1.404 & $<0.001$ & $0.495 \S$ & -1.775 & 1.169 & $<0.001$ & NS \\
\hline $\mathrm{en}_{\mathrm{r}}-\mathrm{en}$ & 0.364 & 1.467 & NS & NS & 0.011 & 1.797 & NS & NS & 1.321 & 2.026 & $<0.001$ & $-0.637 \S$ & 1.477 & 1.816 & 0.003 & NS \\
\hline \multicolumn{17}{|l|}{ Right side } \\
\hline os-or & 0.748 & 1.023 & $<0.001$ & NS & 0.365 & 1.184 & NS & NS & -0.910 & 1.026 & $<0.001$ & NS & -1.369 & 1.075 & $<0.001$ & NS \\
\hline en-ex & -0.141 & 1.491 & NS & NS & 0.014 & 1.745 & NS & NS & -2.124 & 2.206 & $<0.001$ & $0.681 \S$ & -2.469 & 1.535 & $<0.001$ & NS \\
\hline os-or/en-ex & 0.724 & 1.351 & 0.004 & NS & 0.597 & 1.643 & NS & NS & 2.806 & 2.979 & $<0.001$ & $-0.685 \S$ & 2.388 & 2.493 & 0.001 & NS \\
\hline Area & 0.459 & 1.290 & NS & NS & 0.054 & 1.008 & NS & NS & -1.364 & 1.383 & $<0.001$ & $0.584 \S$ & -1.721 & 1.322 & $<0.001$ & NS \\
\hline en-ex vs TH & -0.272 & 1.344 & NS & NS & -0.166 & 1.234 & NS & NS & -1.214 & 1.720 & $<0.001$ & NS & -0.965 & 1.501 & 0.003 & NS \\
\hline os-or vs $\mathrm{TH}$ & 0.561 & 0.982 & 0.002 & NS & 0.433 & 1.158 & NS & NS & 2.009 & 1.291 & $<0.001$ & $-0.536 \S$ & 0.898 & 1.124 & NS & NS \\
\hline os-or vs FH & 0.238 & 1.156 & NS & NS & 0.045 & 1.035 & NS & NS & -3.047 & 1.891 & $<0.001$ & $0.519 \S$ & -1.778 & 1.788 & 0.001 & $0.592 \ddagger$ \\
\hline \multicolumn{17}{|l|}{ Left side } \\
\hline os-or & 0.808 & 1.285 & 0.001 & NS & 0.676 & 1.088 & NS & NS & -0.670 & 1.385 & 0.001 & NS & -0.749 & 1.022 & 0.006 & NS \\
\hline en-ex & -0.864 & 1.259 & $<0.001$ & NS & -0.840 & 1.344 & NS & NS & -1.539 & 1.523 & $<0.001$ & $0.614 \S$ & -1.519 & 1.457 & $<0.001$ & NS \\
\hline os-or/en-ex & 1.362 & 1.441 & $<0.001$ & NS & 1.406 & 1.066 & $<0.001$ & NS & 2.051 & 2.247 & $<0.001$ & $-0.69 \S$ & 2.116 & 2.668 & 0.004 & NS \\
\hline Area & 0.099 & 1.181 & NS & NS & 0.073 & 1.297 & NS & NS & -1.023 & 1.217 & $<0.001$ & $0.38 \ddagger$ & -1.315 & 1.291 & $<0.001$ & NS \\
\hline en-ex vs TH & -0.215 & 1.078 & NS & NS & 0.068 & 0.935 & NS & NS & -0.458 & 1.387 & $<0.001$ & $0.542 \S$ & -0.254 & 1.190 & NS & NS \\
\hline os-or vs $\mathrm{TH}$ & 0.802 & 0.908 & $<0.001$ & $-0.482 \ddagger$ & 0.780 & 1.006 & 0.003 & NS & 1.103 & 1.001 & NS & NS & 0.543 & 1.219 & NS & NS \\
\hline os-or vs FH & 0.658 & 0.956 & NS & NS & 0.778 & 1.241 & $<0.001$ & NS & -2.457 & 1.554 & $<0.001$ & $0.487 \S$ & -1.399 & 1.693 & 0.003 & NS \\
\hline
\end{tabular}

All values are $z$ scores.

*Probability value of paired Student's $t$-test (significance of the $z$ score).

$\dagger$ Correlation coefficient of the $z$ score value versus age; only significant values are reported.

$\ddagger P<0.01$.

$\S P<0.001$.

NS indicates not statistically significant $(P>0.01)$; TH, true horizontal; FH, Frankfort plane.

sex and ethnic group as between-subjects factors: 2-way ANCOVAs for unpaired measurements (biorbital and intercanthal widths; the sex $\times$ group interaction was also computed) and 3-way ANCOVAs for paired measurements (height of the orbit, length of the eye fissure, height-to-length ratio, external orbital surface area, inclinations of the eye fissure and orbit, inclination of the orbit relative to Frankfort plane; within-subject factor: side; the sex $\times$ group, side $\times$ age, side $\times$ group, and side $\times$ sex $\times$ group interactions were also computed).

For all analyses, $P \leq 0.01$ was considered significant.

\section{RESULTS}

The analyzed subjects with DS had a wide range in age, encompassing childhood, adolescence, young, and midadulthood; the mean ages of Italian and North Sudanese subjects were significantly different $(P<0.001,2$-way factorial analysis of variance), whereas no differences were found between sexes $(P>0.01)$. To allow a global assessment, $z$ scores were computed using the mean values of normal, reference individuals of the same age, sex, and ethnicity.

In North Sudanese men and women with DS, almost all $z$ scores were significantly different from 0 (Table $1, P<0.01$ ); the only exceptions were the inclinations of eye fissure and orbit $(P>0.01)$. Intercanthal width was significantly increased, and biorbital width reduced, with smaller right and left eye height, length, and area, when compared with reference subjects. On both sides, the height-to-length ratios were increased. The inclinations of the eye fissure and of the orbit relative to Frankfort plane were reduced, whereas the inclinations of the orbit versus the true horizontal were increased. In North Sudanese women with DS, only $1 z$ score modified significantly with age: negative $z$ scores (values smaller than reference values) tended to normality (0) with growth and aging. In men, more variable patterns were observed, with values that tended toward normality (ex-ex), values that increased with increasing age (en-ex, area), and values that decreased with increasing age (en-en, ratios, os-or vs TH).

In Italian subjects with DS, less than half of the $z$ scores reached significance; women had the lowest number of significant $z$ scores. Overall, when considering the differences that reached significance, in men orbital height was increased, but eye length decreased, with an increased height-to-length ratio relative to reference subjects; the inclinations of the orbit versus the true horizontal were increased. In men, only the inclination of the orbit versus the true horizontal significantly changed with age: in younger subjects, the $z$ scores were mostly positive (values larger than in the reference group) and became nearer to 0 (normal value) with aging. In women, ex-ex distance had a negative $z$ score in younger subjects that became nearer to 0 with aging.

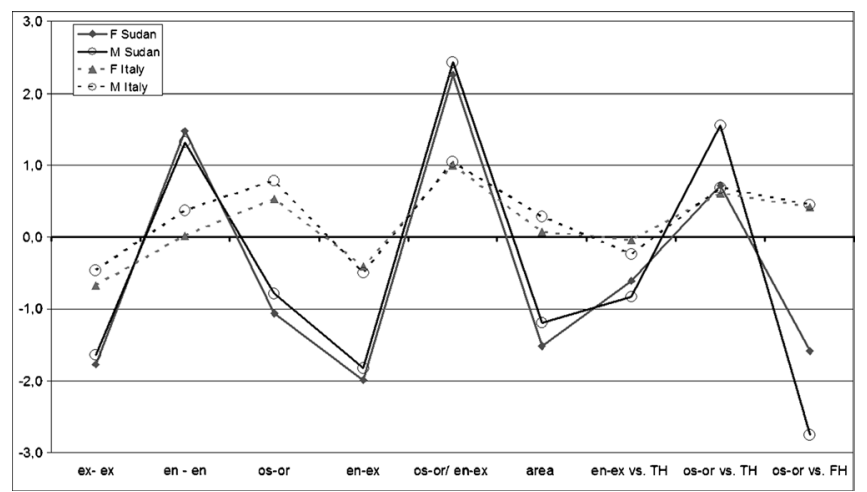

FIGURE 2. Anthropometric pattern profile of subjects with DS. Data are mean $z$ scores in women (closed symbols) and men (open symbols) of Italian (interrupted line) and North Sudanese (continuous lines) ethnic origin. 
TABLE 2. P From the Analyses of Covariance

\begin{tabular}{|c|c|c|c|c|c|c|c|c|c|}
\hline \multirow[b]{2}{*}{ Measurement } & \multicolumn{4}{|c|}{ Between Subjects } & \multicolumn{5}{|c|}{ Within Subjects } \\
\hline & Age & Sex & Group & Sex $\times$ Group & Side & Side $\times$ Age & Side $\times$ Sex & Side $\times$ Group & Side $\times$ Sex $\times$ Group \\
\hline $\mathrm{ex}_{\mathrm{r}}-\mathrm{ex}_{1}$ & 0.005 & NS & NS & NS & - & - & - & - & - \\
\hline $\mathrm{en}_{\mathrm{r}}-\mathrm{en}_{1}$ & 0.002 & NS & NS & NS & - & - & - & - & - \\
\hline os-or & NS & NS & $<0.001$ & NS & NS & NS & NS & NS & NS \\
\hline en-ex & $<0.001$ & NS & NS & NS & NS & NS & NS & $<0.001$ & NS \\
\hline os-or/ en-ex & $<0.001$ & NS & NS & NS & NS & NS & NS & .007 & NS \\
\hline Area & NS & NS & $<0.001$ & NS & NS & NS & NS & NS & NS \\
\hline en-ex vs TH & 0.008 & NS & NS & NS & NS & NS & NS & 0.005 & NS \\
\hline os-or vs TH & $<0.001$ & NS & NS & NS & NS & NS & NS & $<0.001$ & NS \\
\hline os-or vs FH & 0.003 & NS & $<0.001$ & NS & NS & NS & NS & NS & NS \\
\hline
\end{tabular}

Degrees of freedom: 1,112 for all comparisons.

NS indicates not significant $(P>0.01)$; TH, true horizontal; FH, Frankfort plane.

Both ethnic groups and both sexes had almost the same discrepancies from the controls, but in Italian subjects, most of the $z$ scores were nearer to 0 than in North Sudanese subjects (Fig. 2). To assess if the discrepancies between control subjects and subjects with DS were the same or differed in the 2 ethnic groups, ANCOVAs were performed, using age as covariate (Table 2). For all measurements but soft tissue orbital height and area, a significant $(P<0.01)$ effect of age was found. No significant effects of sex were found. A significant effect of ethnic group was found for orbital height, area, and orbital inclination versus Frankfort plane: North Sudanese subjects with DS had smaller values than reference subjects, whereas the opposite was found in Italian subjects.

All paired measurements had similar discrepancies on both sides, without significant differences (3-way ANCOVAs, $P>0.01$ ). Scanty significant side $\times$ group interactions were found: in all occasions, the $z$ scores between the 2 ethnic groups were more similar on the left than on the right side.

\section{DISCUSSION}

In the present investigation, some anthropometric measurements performed on the soft tissues of the orbital region have been found to differ between control subjects and subjects with DS. The discrepancies between control subjects and patients were larger in North Sudanese persons than in Italian ones, and in some occasions, a significant effect of ethnic group was found, with opposite results: orbital height, area, and orbital inclination versus Frankfort plane were smaller in North Sudanese subjects with DS than in reference subjects, whereas the opposite was found in Italian subjects. The differences in age and sex composition of the 2 groups were taken into account using ANCOVAs.

Previous investigations performed on control subjects found that the dimensions and positions of the orbital soft tissues were sexually dimorphic; were modified between childhood, adolescence, and young adulthood, and even after young adulthood into the eighth decade of life; and had some ethnic-related characteristics. ${ }^{2,4-7}$

A detailed comparison of the present results with literature data is not possible, because the characteristics of the soft tissues of the orbital region were insufficiently analyzed in DS subjects, and no data on North Sudanese subjects were published. Sudan, the largest country in Africa, has a multiethnic population; the 3 major ethnic groups are those of Arab descent in the north, Nilotic tribes in the south, and West African tribes in the region of Darfur and Eastern Tribes; each tribe has marked facial morphologic differences. Previous studies on the facial phenotype of North Sudanese subjects with DS, as compared with control subjects, assessed only the nasolabial and auricular regions. ${ }^{20,21}$ Overall, published reports showed that the nasal characteristics of North Sudanese subjects with DS well matched those found in white subjects, with a smaller nose with reduced vertical and anteroposterior dimensions, but with increased horizontal dimensions. ${ }^{20}$ Similar results were also obtained for the discrepancies in ear characteristics: in both ethnic groups, subjects with DS had smaller ear length, width, and area; larger ear width-to-ear length ratios; more prominent ears; and larger asymmetry in the position of paired ear landmarks than subjects with normal karyotype. ${ }^{21}$ In contrast, lip modifications in North Sudanese subjects with DS (reduced total cutaneous volume and dimensions but increased vermilion areas and relevant dimensions) were less marked than in white subjects with DS. ${ }^{20}$

In contrast with the results found for the labial region, soft tissue orbital modifications were more marked in North Sudanese than in white subjects with DS: for instance, reductions in biorbital width and increments in intercanthal width were found in all ethnic and sex groups, but they reached the statistical significance only in North Sudanese persons.

In Italian subjects with DS, the limited discrepancies found in the soft tissues of the orbital region were in accord with the results reported by Pommier et al, ${ }^{16}$ who did not find marked skeletal orbital anomalies in their three-dimensional computed tomographic study of fetuses with DS analyzed between 17 and 25 weeks of gestational age. They tentatively explained their data because no central nervous system alterations are classically associated with DS. Indeed, investigations on other developmental disorders found a significant relationship between facial phenotype and central nervous system alterations. ${ }^{1,2,22}$

In fetuses with DS, increased skeletal orbital height (in accord with the current result in Italian subjects but in contrast with that in North Sudanese ones) and interocular distance (in good accord with the current findings, especially in North Sudanese subjects) were found. ${ }^{16}$ In contrast, in a three-dimensional ultrasonographic study, Roelfsema et $\mathrm{al}^{17}$ found that a 29 -week-old fetus with DS had significantly ( $>2$ SDs) reduced bony orbital dimensions (interocular and outer ocular distances) when compared with normal fetuses of the same gestational age. For both studies, no information about the ethnicity of their fetuses was reported, and only further investigations may help in better understanding of these contrasting data.

In Italian subjects, current $z$ scores for biorbital and intercanthal widths, eye fissure length, and inclination are in good accord with data reported for white North American ${ }^{11,12}$ and Croatian ${ }^{9}$ subjects with DS analyzed with conventional anthropometry.

In Italian subjects with DS, almost no significant age-related variations in the $z$ scores were found, in accord with previous reports 
on other craniofacial characteristics. ${ }^{15,18}$ One explanation may be the relatively reduced age range (from 13 to 52 years). Indeed, Farkas et $\mathrm{al}^{12}$ found limited age-related variations in the frequency of normal (within 2 SDs) and abnormal ( $>2$ SDs) $z$ scores of the orbital region in DS subjects between early childhood (1-5 years) and young adulthood (16-35 years). In contrast, in North Sudanese men, a significant effect of age was found for $75 \%$ of the analyzed measurements; in several occasions, a trend toward a normalization of the values ( $z$ scores nearer to 0 ) was found. Indeed, in some developmental disorders, facial anomalies have been reported to diminish with age. ${ }^{1}$ Considering the cross-sectional nature of the current study (different subjects were analyzed at different ages), ${ }^{7}$ a different explanation may be that the analyzed children and adults had a different phenotype and that the children with greater $z$ scores were not measured in their young and mid adulthood.

No significant differences between male and female $z$ scores were found: for North Sudanese subjects, this finding parallels what was found in the nasolabial and auricular areas, with similar patterns of discrepancies in the 2 sexes. $^{20,21}$ For white subjects, previous investigations found no significant sex-related differences. ${ }^{9,14,15,18}$

Sample composition is one of the limitations of the current study: for both ethnic groups, convenience subjects were analyzed, with different age ranges between them. In addition, Italian subjects were all athletes attending Special Olympics game, and they all had practiced 1 or more sports. ${ }^{14,15,18,19}$ In contrast, Sudanese subjects were all attending special needs schools and did not attend any specific sports or physical activity program. ${ }^{20,21}$ Indeed, the possible effect of a better general body muscular tone has already been proposed to explain some differences in the nasolabial area, ${ }^{14,15,18}$ but it seems of lesser importance for the soft tissues of the orbital region.

A further limitation is that 2 different instruments/methods were used for data collection by 2 different teams, and the 2 procedures have never been tested one against the other. Therefore, no actual measurements were analyzed, but all values were converted into $z$ scores using reference values collected under the same conditions. $z$ Scores were also used because different numbers of men and women were measured in the 2 ethnic groups, and the age composition of the groups was not matched. Also, the reduced number of subjects with DS impeded separate sex- and age-related analyses. Overall, this procedure allows to partially control for the mixed composition of the DS group and permitted to assess if the discrepancies between control subjects and subjects with DS are the same or differ in the 2 ethnic groups. The procedure has already been used in previous investigations on the craniofacial characteristics of individuals with genetic or acquired alterations. ${ }^{9-13,17,20,21}$

In conclusion, we found that the orbital soft tissues of North Sudanese subjects with DS differed from those of their reference subjects of the same age, sex, and ethnicity, but this was only partially true for Italian subjects. Also, the 2 ethnic groups had different alterations in their soft tissue orbital regions, even after correcting for the age and sex composition of the sample. The alterations were influenced by age but did not differ between men and women, which had less significant discrepancies from the norm than men. Indeed, this last effect may be directly related to the reduced number of analyzed women in both ethnic groups, and it should be further assessed with larger samples.

\section{REFERENCES}

1. Mutsvangwa TE, Meintjes EM, Viljoen DL, et al. Morphometric analysis and classification of the facial phenotype associated with fetal alcohol syndrome in 5- and 12-year-old children. Am J Med Genet A 2010;152A:32-41

2. Moore ES, Ward RE, Wetherill LF, et al. Unique facial features distinguish fetal alcohol syndrome patients and controls in diverse ethnic populations. Alcohol Clin Exp Res 2007;31:1707-1713

3. Weaver AA, Loftis KL, Tan JC, et al. CT based three-dimensional measurement of orbit and eye anthropometry. Invest Ophthalmol Vis Sci 2010;51:4892-4897

4. Kunjur J, Sabesan T, Ilankovan V. Anthropometric analysis of eyebrows and eyelids: an inter-racial study. Br J Oral Maxillofac Surg 2006;44:89-93

5. Park DH, Choi WS, Yoon SH, et al. Anthropometry of Asian eyelids by age. Plast Reconstr Surg 2008;121:1405-1413

6. Price KM, Gupta PK, Woodward JA, et al. Eyebrow and eyelid dimensions: an anthropometric analysis of African Americans and Caucasians. Plast Reconstr Surg 2009;124:615-623

7. Sforza C, Grandi G, Catti F, et al. Age- and sex-related changes in the soft tissues of the orbital region. Forensic Sci Int 2009;185:115.e1-115.e8

8. OMIM. Online Mendelian Inheritance in Man. Available at: http://www.ncbi.nlm.nih.gov/entrez/query.fcgi?db=OMIM. Accessed December 11, 2010

9. Bagic I, Verzak Z. Craniofacial anthropometric analysis in Down's syndrome patients. Coll Antropol 2003;27:23-30

10. Farkas LG, Katic MJ, Forrest CR. Surface anatomy of the face in Down's syndrome: anthropometric proportion indices in the craniofacial regions. J Craniofac Surg 2001;12:519-526

11. Farkas LG, Katic MJ, Forrest CR, et al. Surface anatomy of the face in Down's syndrome: linear and angular measurements in the craniofacial regions. J Craniofac Surg 2001;12:373-379

12. Farkas LG, Katic MJ, Forrest CR. Age-related changes in anthropometric measurements in the craniofacial regions and in height in Down's syndrome. J Craniofac Surg 2002;13:614-622

13. Farkas LG, Katic MJ, Forrest CR. Surface anatomy of the face in Down's syndrome: age-related changes of anthropometric proportion indices in the craniofacial regions. J Craniofac Surg 2002;13:368-374

14. Ferrario VF, Dellavia C, Zanotti G, et al. Soft tissue facial anthropometry in Down syndrome subjects. J Craniofac Surg 2004;15:528-532

15. Ferrario VF, Dellavia C, Serrao G, et al. Soft tissue facial angles in Down's syndrome subjects: a three-dimensional non-invasive study. Eur J Orthod 2005;27:355-362

16. Pommier S, Adalian $\mathrm{P}$, Gaudart J, et al. Fetal age estimation using orbital measurements: 3D CT-scan study including the effects of trisomy 21. J Forensic Sci 2009;54:7-12

17. Roelfsema NM, Hop WC, van Adrichem LN, et al. Craniofacial variability index in utero: a three-dimensional ultrasound study. Ultrasound Obstet Gynecol 2007;29:258-264

18. Sforza C, Dellavia C, Zanotti G, et al. Soft tissue facial areas and volumes in subjects with Down syndrome. Am J Med Genet A 2004;130A:234-239

19. Sforza C, Dellavia C, Tartaglia GM, et al. Morphometry of the ear in Down's syndrome subjects. A three-dimensional computerized assessment. Int J Oral Maxillofac Surg 2005;34:480-486

20. Sforza C, Elamin F, Rosati R, et al. Three-dimensional assessment of nose and lip morphology in North Sudanese subjects with Down syndrome. Angle Orthod 2001;81:109-116

21. Sforza C, Elamin F, Rosati R, et al. Morphometry of the ear in north Sudanese subjects with Down syndrome. A three-dimensional computerized assessment. J Craniofac Surg 2011;22:297-301

22. Hammond P, Forster-Gibson C, Chudley AE, et al. Face-brain asymmetry in autism spectrum disorders. Mol Psychiatry 2008;13:614-623

23. Ferrario VF, Sforza C, Poggio CE, et al. Preliminary evaluation of an electromagnetic three-dimensional digitizer in facial anthropometry. Cleft Palate Craniofac J 1998;35:9-15 